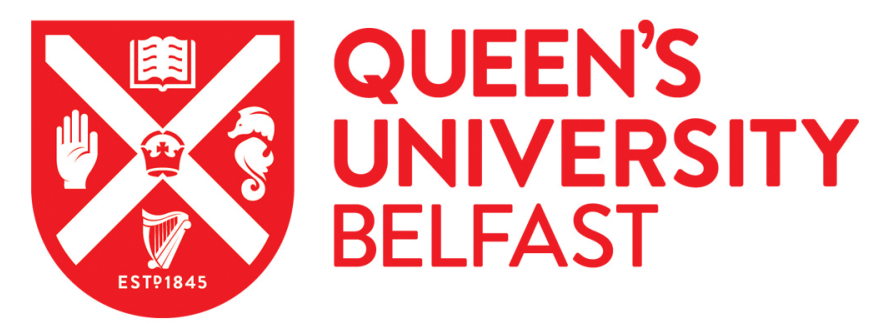

\title{
Traumatic brain injury and co-occurring problems in prison populations: a systematic review.
}

O'Rourke, C., Linden, M. A., Lohan, M., \& Bates-Gaston, J. (2016). Traumatic brain injury and co-occurring problems in prison populations: a systematic review. Brain Injury, 30(7), 839-854.

https://doi.org/10.3109/02699052.2016.1146967

\section{Published in:}

Brain Injury

\section{Document Version:}

Peer reviewed version

\section{Queen's University Belfast - Research Portal:}

Link to publication record in Queen's University Belfast Research Portal

\section{Publisher rights}

Copyright 2016 Taylor and Francis

This is an Accepted Manuscript of an article published by Taylor \& Francis in Brain Injury on 18 Apr 2016, available online at: http://www.tandfonline.com/doi/full/10.3109/02699052.2016.1146967

\section{General rights}

Copyright for the publications made accessible via the Queen's University Belfast Research Portal is retained by the author(s) and / or other copyright owners and it is a condition of accessing these publications that users recognise and abide by the legal requirements associated with these rights.

Take down policy

The Research Portal is Queen's institutional repository that provides access to Queen's research output. Every effort has been made to ensure that content in the Research Portal does not infringe any person's rights, or applicable UK laws. If you discover content in the Research Portal that you believe breaches copyright or violates any law, please contact openaccess@qub.ac.uk. 


\section{Traumatic brain injury and co-occurring problems in prison populations: a systematic review.}

Conall O’Rourke ${ }^{1}$ BSc, Mark A. Linden ${ }^{1} \mathrm{PhD}$, Maria Lohan ${ }^{1} \mathrm{PhD}$ and Jackie Bates-Gaston ${ }^{2}$ $\mathrm{PhD}$.

1. School of Nursing \& Midwifery, The Queen's University of Belfast, United Kingdom. 2. Northern Ireland Prison Service Headquarters, Dundonald House, Stormont Estate, United Kingdom.

Running head: TBI in prisoners: a systematic review

Funding received: This research was supported by the Department of Education and Learning, Northern Ireland

Conflicts of interest: The authors report no conflicts of interest.

Accepted for publication in Brain Injury 2015 


\begin{abstract}
Background: A growing body of epidemiological research suggests high rates of traumatic brain injury (TBI) in prisoners. The aim of this review is to systematically explore the literature surrounding the rates of $\mathrm{TBI}$ and their co-occurrences in a prison population.

Methods: Six electronic databases were systematically searched for articles published between 1980 and 2014. Studies were screened for inclusion based on predetermined criteria by two researchers who independently performed data extraction. Study quality was appraised based on a modified quality assessment tool.
\end{abstract}

Results: Twenty six studies were included in this review. Quality assessment ranged from $20 \%$ (poor) to $80 \%$ (good) with an overall average of $60 \%$. Twenty four papers included TBI prevalence rates, which ranged from $5.69 \%-88 \%$. Seventeen studies explored co-occurring factors including rates of aggression $(n=7)$, substance abuse $(n=9)$, anxiety and depression $(n=5)$, neurocognitive deficits $(n=4)$, and psychiatric conditions $(n=3)$.

Conclusions: The high degree of variation in TBI rates may be attributed to the inconsistent way in which TBI was measured with only seven studies using valid and reliable screening tools. Additionally, gaps in the literature surrounding personality outcomes in prisoners with TBI, female prisoners with $\mathrm{TBI}$, and qualitative outcomes were found.

Key words: Traumatic brain injury, prisoners, incarceration, systematic review 


\section{Introduction}

Traumatic brain injury (TBI) is recognised as one of the leading causes of death and disability in young people and adults (1). Statistics from the USA estimate TBI rates to be around 533 per 100,000 (2). Australian population studies support similar numbers, with TBI rates of around 226 per 100000 observed (3,4). A recent meta-analysis by Tagliaferri et al. (4) revealed equally high European rates ranging from 235 per 100000 to 546 per 100000 in some regions.

The vast majority of TBIs fall into the mild range and usually result in a mixture of somatic (headaches and dizziness), cognitive (forgetfulness/poor concentration) and behavioural (fatigue and irritability) symptoms which generally dissipate within a few weeks (5). More severe injuries may lead to significant neurocognitive and developmental difficulties, depending on the affected region of the brain (6). Frontal lobe damage can impact on executive functions and behavioural regulation while insults to the temporal lobes can lead to memory and learning difficulties (5). Diffuse axonal injuries which take the form of white matter lesions can also impair the speed of information processing (5). Such injuries can manifest in a myriad of ways including emotional and personality changes $(7,8)$, impaired social function $(9,10)$, and aggression $(11,12)$. Without proper identification and treatment, executive function deficits such as these may lead to increased antisocial and even criminal behaviour (13).

Large scale estimates from epidemiological studies have identified several populations at high risk of TBI. Specifically, children aged 0 to 14 years account for thirty percent of the TBIs per year in the USA (2). Sex differences are also widely reported with males aged 14-25 up to four times more likely to suffer a TBI than females of the same age (14). Equally worrying is the increased risk of arrest following TBI, observed in a recent longitudinal study by Elbogen et al. (15). They examined data from the Traumatic Brain Injury Model System (TBIMS) National Database, representing participants 
aged 16 years or over who had experienced moderate to severe TBI. Researchers followed up participants at 1, 2, and 5 years to assess post-TBI criminal arrest. They found that participants who received brain injuries with loss of consciousness (LOC) lasting under 24 hours were at greater risk of future criminal arrest. While no data was available to indicate the nature of the crimes (violent vs non-violent) the results are suggestive of a relationship between TBI and criminal behaviour. It should also be noted that additional risk factors independent of injury were also identified (i.e. young, male, single, low education) and the authors highlight the need for additional research to explore these and other factors such as premorbid criminal behaviour and substance abuse.

The study above is indicative of the growing body of literature surrounding TBI in incarcerated groups, with past meta-analyses consistently reporting high levels of TBI in both adult (16), and juvenile offenders (17). Shiroma et al. (16) analysed twenty studies comparing TBI prevalence, gender proportion, case definition of TBI, and method of determining TBI. They estimated TBI prevalence to be $60.25 \%$ in incarcerated adults, but when analysis was restricted to only include cases of TBI with loss of consciousness (TBILOC) this rate dropped to $50.19 \%$. Likewise, when comparing between males and females, TBI rates of $64.41 \%$ and $69.98 \%$ dropped to $59.31 \%$ and $55.28 \%$ when only TBIs that resulted in a loss of consciousness were included. Using broad offender definitions they included studies focusing on specific subsets of the general prison population (i.e. death row inmates, murderers, maximum security psychiatric patients) and several non-incarcerated populations (i.e. community support group, court ordered rehabilitation programs) making the results difficult to extrapolate to general prison populations.

Among juvenile offenders Farrer et al. (17) found lower TBI prevalence rates (30.6\%), but when compared to control groups these offenders were 3.37 times more likely to have a brain injury. A recent systematic review by Hughes et al. (18) found TBI prevalence in this group to be significantly 
higher with between $49.7-71.2 \%$ of young people in custody reporting a past brain injury. As with previous studies, when injury was limited to LOC, rates fell to between $16.5 \%$ and $49 \%$. It should also be noted that Farrer et al. (17) included juveniles in community youth offending teams (YOT) while Hughes et al. (18) focused specifically on young offenders in custody which may explain the variance in results.

While these reviews broadly discuss TBI in offending populations their focus was on either young offenders or included offenders from non-prison settings. We approached this review with the aim of systematically analysing the literature surrounding TBI in adult prison populations with a focus on both prevalence and co-occurring problems.

\section{Method}

\section{Electronic sources and search strategy}

Six databases, CINAHL, EMBASE, Medline, PsycInfo, PubMed, and Web of Science, were systematically searched between $26 / 01 / 2015$ to $05 / 02 / 2015$. Databases were chosen based on their access to a wide range of international articles across many disciplines. A brief 'scoping review' was conducted, which involved identifying literature relevant to the review question and extracting commonly used search terms. Several studies with relevance to the aim of the review were identified. Keywords were then extracted from these articles and reviewed for inclusion in the search strategy to increase the likelihood of identifying other relevant articles. The search terms included were as follows;

Head injur* OR Brain injur* OR TBI OR ABI OR Concussion OR Right Hemisphere injur* OR Left Hemisphere Injur* OR Cumulative Trauma Disorders OR Cerebrovascular Trauma OR Craniocerebral Trauma OR Brain Edema OR Diffuse Axonal Injury OR Coma OR Cerebral Hemorrhage. 
AND

Prisoner* OR Inmate* OR Criminal* OR Offender OR Convict OR Felon OR Incarcerated OR Detainees

OR Public Offenders OR Prison OR Jail OR Penitentiary OR Correctional Facilit* OR Correctional Health Services OR Imprisoned OR recidivism.

Where possible, specific publication criteria were set to ensure all articles were in English, and published in peer reviewed journals. Limits on publication date were set at 1980 to ensure a fair representation of current prisoner populations with TBI. Articles from each database were then combined and duplicates were removed. COR and ML screened articles by title and abstract applying the following inclusion and exclusion criteria. A flow chart summary of the search strategy and exclusion of papers can be found in figure 1.

\section{Inclusion and exclusion criteria}

Peer-reviewed empirical studies which provided rates of TBI in adult prisoner populations (age 18 and older) were included. As no resources were available for translation, only papers published in English were accepted in the review. No restrictions were placed on the design of studies.

As the review focused on the general prison population, studies examining other populations e.g. death-row, psychiatric, or elderly inmates, were excluded. We also excluded papers in which the source of offenders was unclear or in which participants were recruited from community correction programs or attending court mandated rehabilitation programs.

\section{Data extraction and quality appraisal}

Data extraction and quality appraisal were then performed on all eligible articles by COR and ML, allowing for discussion and comparison of differences in coding. In the case of disagreement a third reviewer (JBG) was available to arbitrate. Quality appraisal of all identified studies was conducted for 
the purpose of assessing the standard of research produced in this area and was measured using an adapted quality assessment tool produced by the National Institute of Health (19). The original checklist consisted of 14 criteria to measure both the internal and external validity of observational, cohort and cross sectional studies. The researchers decided to split question four which asked whether participants were selected from the same or similar populations and whether inclusion and exclusion criteria were uniformly applied to all participants, into two separate questions. Focus was placed on the research question, study population, outcome and exposure measures for each study, and single scores were allocated for each of the criteria. Individual criteria were then totalled to give each study a score between $0-15$. Studies scoring 7 or above were considered acceptable and those scoring below this threshold $(n=3)$ were considered poor.

\section{Data analysis}

Due to the heterogeneity of measurement it was not possible to conduct a meta-analysis. A narrative description of the studies was deemed to be most appropriate for the aims of the review.

\section{Results}

\section{Overview of included studies}

We included 26 studies published between 1992 and 2014 in this review. Fourteen studies were undertaken in the USA, five in Australia, two in Canada, two in the UK, two in New Zealand, and one in Spain. Sample sizes ranged from 38 (20) to 1055 (21) participants with the exception of the longitudinal Shiroma et al. (22) study in which 21,610 participants were analysed. All studies were quantitative and no qualitative studies meeting the eligibility criteria were found.

\section{Framework for analysis}


Studies were divided based on their inclusion of either; a measure of TBI rates ( $n=24)$, or a measure of co-occurring factors ( $n=17)$. The extracted data for all included studies can be found in table 1 .

\section{Insert figure 1 here}

\section{Insert table 1 here}

\section{Quality assessment}

The results of the quality assessment are found in Table 1, presented as a single score between 0-15. The overall average quality of included papers was 9 with scores ranging from 3 to 12 . Templer et al. (21) and Morrell et al. (35) failed to achieve above a score of 5 having inadequately reported on their population, criteria for inclusion, the recruitment procedure, and the independent and dependent variables.

The majority of studies included a clearly stated research question/objective, evident in $96 \%$ ( $n=25$ ) of cases. Few studies reported a justification of sample size (9\%) $(n=2)$ and just over one third of studies utilised valid and reliable exposure measures $(42 \%)(n=11)$ with slightly more using validated outcome measures $(46 \%)(n=12)$.

\section{Rates of TBI in prison populations}

While not all papers reported TBI rates within their populations, only one study failed to offer a clear definition of TBI (32). Variation was evident both in the definitions used for TBI and the rates reported between studies, with figures for TBI ranging from $5.69 \%$ (22) to $88 \%$ (29).

A number of studies $(n=9)$ categorised injuries as either mild, moderate, or severe (22$24,27,29,37,38,43,45)$ while others $(n=10)$ used LOC to categorise severity $(25,28,30,31,34-36,40-$ 
42). Three studies focused solely on one type of injury (TBILOC $(21,26))$, with Suchy et al. $(20)$ limiting inclusion to $\mathrm{mTBI}$ and excluding participants who reported more than two instances of LOC in their lifetime. Walker et al. (44) categorised severity by either multiple injury, single injury, or no injury. Ross and Hoaken (39) divided injuries into either concussions or serious brain injury (defined as prior head or brain injury not categorised as a concussion), while Kavanagh et al. (32) recorded all injuries under a single label (head injury). Finally, León-Carrion and Ramos (33) defined participants as having either a brain injury (any type of blow/injury to the head), or severe brain injury (a blow to the head with consequences). It should be noted that no description of these consequences was provided, creating confusion as to the severity of such blows to the head.

The nature of the measures used for screening TBI also varied considerably with only seven studies using clearly validated tools to assess historic TBI. The most popular of these, applied in five studies, was the OSU-TBI-ID. Bogner and Corrigan (25) Fishbein et al. (31), and Ferguson et al. (30) utilised the full measure, while Ray et al. (38) adopted a modified shortened version for use in their sample. Similarly high rates were reported in the studies employing the full tool; $78 \%$ in the Bogner and Corrigan (25), 67.8\% in the Fishbein et al. (31), and 67\% in the Ferguson et al. (30) study. The shortened version however, reported almost half this level (35.7\%). Corrigan et al. (28) sourced their prison sample from a previous study by Bogner and Corrigan (25) and their use of the OSU-TBI-ID yielded rates of TBI equal to those of the earlier study (78\%).

The remaining two studies which used validated screening tools were Diamond et al. (29) and Pitman et al. (37). The primary aim of the Diamond et al. (29) study was to assess the validity of TBIQ as a screening tool for past TBI. In addition to supporting its reliability, they reported a TBI rate of $88 \%$ among their mixed gender prison sample. Pitman et al. (37) was the only study to utilise the Brain Injury Screening Index (BISI) on their male prison population. While their primary aim was to 
examine the association between TBI and cognitive performance, their initial screening of eligible participants revealed that $47 \%$ sustained past TBIs.

Although none of the remaining eighteen studies used clearly validated tools, there were overlaps observed in the style of investigation. Slaughter et al. (43) utilised a simple two question format to assess past TBI; '(1) Have you ever had an injury to the head, which knocked you out or at least left you dazed, confused, or disoriented? (2) How many injuries like this have you had over your lifetime?' ((43), pg 733). This was adapted from criteria defined by the Head Injury Interdisciplinary Special Interest Group of the American Congress of Rehabilitation Medicine (46) and subsequently applied as a severity measure of past injuries. Their study revealed overall TBI rates of $87 \%$ among their sample of male and female prisoners. Subsequent studies $(27,36,41,42)$, employed the same two questions to screen for TBI in their own prison samples. Both studies by Schofield et al. $(41,42)$ used the same population, therefore the rate of TBI was identical for both groups (82\%). The Perkes et al. (36) study, of which Schofield was an author, also reported the rate of TBI as $82 \%$, while Colantonio et al. (27) reported a TBI rate of $43.4 \%$ among their sample. While all studies that employed this style of questioning reported significant rates of TBI, there were no sources of research supporting the validity of this style of measurement.

Among the other methods used, Brewer-Smyth et al. (26) was the only study to employ a clinical nurse specialist to assess past TBI, finding that $42 \%$ of the sample endorsed at least one TBILOC. Schofield et al. (40) compared self-reported TBI to hospital records with the aim of assessing the validity of prisoner self-report. The authors used an unvalidated measure to assess self-reported past TBI, revealing a rate of $82 \%(n=164)$ among 200 inmates. A total of 420 individual injuries were recorded, of which only 112 resulted in attendance at a hospital accessible by the researchers. 83\% (93/112) of these reported TBIs had medical records available of which 70\% (78/112) were validated. 
Shiroma et al. (22) utilised a medical database to assess past TBI limiting their results to medically attended TBIs. This resulted in a much lower rate of TBI for both their male (5.65\%) and female (6.22\%) samples.

Merbitz et al. (34) and Morell et al. (35) both screened for the presence of TBI using an interview format. Merbitz et al. (34) screened 129 prisoners by interview, conducted by a staff psychologist. The rate of TBI was reported as $31.7 \%$, while Morrell et al. (35), using the same interview format, reported TBI rates of $24.9 \%$ among 1000 inmates. The interview format produced much lower levels of self-reported TBI compared with more recent papers employing questionnaires and screening tools. The oldest included study, Templer et al. (21), utilised a brain injury survey to assess for TBI among their sample, which comprised of; male prisoners, college students, college American football players, and psychology students. The survey included one question assessing whether participants had ever had a head injury that led to LOC and a follow-up requesting participants to report the number and details of injuries sustained. In their sample of male prisoners $35.7 \%$ reported sustaining one or more injuries.

Among the remaining papers Williams et al. (45) included only one question 'Have you ever had a head injury or been concussed (knocked out) for a period of time?' coupled with additional followups 'How many times have you been knocked out?' 'Please give details...' ((45), pg 1185). Other studies, such as Walker et al. (44) offered multiple, more specific questions '(a) Have you ever been in an auto accident and received a head injury; (b) Were you ever knocked out in a fight; (c) Have you ever had a head injury from an accident at work or other event; (d) Have you ever had a head injury resulting from a bike/motorcycle accident; and (e) Do you believe you have received a head injury as a child from an accident or from abuse by a parent' ((44), pg 347). The authors then grouped injuries into three categories; (a) multiple brain injury (defined as those reporting two or 
more head injuries); (b) one brain injury (defined as those reporting only one head injury); and (c) no brain injury (defined as those not sustaining a head injury). Results varied between these two approaches, with Williams et al. (45) reporting a TBI rate of $60.7 \%$ compared with $35.7 \%$ reported by Walker et al. (44).

\section{Co-occurring problems}

Seventeen studies explored a range of conditions present among prisoners with TBI. Five studies tested for an interaction between depression/anxiety and TBI $(29,37,41,42,44)$, while nine studies explored the co-occurrence of drug and alcohol abuse $(23,27-29,31,36,37,42,44)$. Aggression and anger were examined in seven studies $(26,29,31,33,37,41,43)$. Psychiatric conditions were explored in three studies $(38,42,43)$, and four studies examined neurocognitive deficits in the context of TBI $(20,24,28,37)$.

\section{Neurocognitive deficits}

Barnfield and Leathem (24) compared 50 prisoners with TBI to normative scores across several neuropsychological measures exploring short and long term verbal and visual memory, information processing, motor speed and co-ordination, executive functioning, and malingering. Results showed that participants with TBI performed significantly lower on a number of short-term auditory-verbal memory and executive function scales. Mean performance on tests of mentally processing, categorising, and conceptualising information in long term memory was also 1.33 standard deviations below the norm.

Pitman et al. (37) explored the cognitive performance of $129 \mathrm{TBI}$ and 50 control prisoners on a range of questionnaires and neuropsychological tests, including test of premorbid functioning, the WASI 
(47), the BADS (48), and the RBANS (49). Participants with TBI performed significantly worse on the RBANS, BADS, and WASI, while past injury correlated negatively with the difference between current WASI and premorbid IQ.

Corrigan et al. (28) divided 257 participants (all of which had past TBI) by common characteristics of lifetime history e.g. number of injuries at various ages, the number of clinical symptoms per injury, the cause of injury etc. They used both the Processing Speed and Working Memory indices from the WAIS-III (50) along with the Alertness Scale of the SIP (51) and the disinhibition subscale of the FrSBe (52) to assess cognitive performance. Clusters defined by early TBI (between 6-10 years old) and serious adult TBI had the slowest information processing speed and the greatest number of cognitive complaints.

Suchy et al. (20) was the only study to focus solely on mTBI. They compared 19 prisoners with injury and 19 prisoners without injury on a range of neuropsychological measures including subscales of the DKEFS (53), the WAIS-III, the SILS (54), and the BDS-EV (55) battery. No significant differences were found between the groups on any measure with the exception of the BDS-EV which measured reaction to novelty. Participants with $\mathrm{mTBI}$ exhibited a significantly larger novelty effect showing a greater sensitivity to new stimuli.

Finally, while Ross and Hoaken (39) compared the rate of TBI in first time and repeat offenders they failed to include this as a factor in their ANCOVA of executive function measures meaning it was impossible to conclude whether executive function deficits co-occurred with TBI.

\section{Drug/alcohol abuse}

Walker et al. (44) sought to measure the co-occurrence of drug use with TBI. They recruited participants with past drug use under the hypothesis that drug users with comorbid TBI would 
present with both significantly more health problems and more serious drug use. Using the ASI (56) they found that TBI was significantly associated with the type and frequency of use in the 12 months preceding incarceration. Additionally, prisoners with one or more brain injuries reported significantly higher alcohol use prior to incarceration.

Fishbein et al. (31) explored the co-occurrence of TBI and drug and alcohol use through a modified substance abuse scale using a single question covering historic alcohol consumption patterns. When participants with TBI were compared to those without, they found significantly more participants without TBI reported never using drugs and had lower levels of alcohol consumption. High drug use was also evident in the sample of prisoners with TBI examined in the Barnfield and Leathem (23) study. The authors also noted that it was not possible to form separate TBI and substance abuse groups for comparison due to the high rate of comorbidity.

Perkes et al. (36) compared drug and alcohol abuse in a sample of male prisoners with TBI and a matched community control group. Using the AUDIT (57) and specific drug related questions they reported that TBI correlated with both recent alcohol use/misuse and recent illicit drug use. Similarly, Schofield et al. (42) explored drug and alcohol misuse through the AUDIT alongside specific drug use questions, finding that prisoners who used illicit drugs daily were 2.5 times more likely to have a TBI. Diamond et al. (29) also found significantly higher rates of historic substance abuse among offenders with TBI.

Not all studies reported statistically significant findings. Colantonio et al. (27) utilised a short form of a computerised substance abuse measure to examine early life experience among inmates with TBI. While they found that men with TBI reported consuming alcohol at an earlier age, no statistically significant differences were found when past TBI was compared to age at first drug use. Corrigan et al. (28) included the Alcohol Use and Drug Use composite scores from the ASI (56) in their cluster 
analysis. They found a negative relationship between alcohol and drug severity for the cluster defined by early TBI (aged 6-10), meaning that greater severity of alcohol use disorder was associated with lower severity of drug use. No explanation was provided for why this occurred. Finally, Pitman et al. (37) showed no statistically significant differences in drug and alcohol misuse between prisoners with and without TBI.

\section{Anxiety/depression}

Both Schofield et al. studies $(41,42)$ measured anxiety and depression using either the RDS $(58)$ or a questionnaire listing possible side effects of TBI. In addition to reporting anxiety as an unresolved effect among $22 \%$ of their sample with TBI, they reported that four or more TBIs and TBILOC were most commonly associated with prisoners who scored positively for major depression. Pitman et al. (37) found similar trends using the BAI (59) and BDI (60) inventories. Participants with past TBI performed significantly worse on these measures when compared to prisoners without injury. Furthermore, Diamond et al. (29) used both a measure of depression, and a personality/psychopathology questionnaire to assess anxiety in American prisoners. They found that mean scores on the depression index were higher for prisoners with one or more TBIs when compared to those without injury. Finally, Walker et al. (44) found higher levels of anxiety, mental health problems, and symptoms of depression in prisoners with two or more brain injuries as measured by the ASI (56). Prisoners with TBI were also more likely to report a history of suicidal ideation and suicide attempts than the group without injury.

\section{Aggression/violence}

Slaughter et al. (43) employed the BAAQ (61) to examine anger and aggression, finding significantly higher rates among prisoners with TBI when compared to prisoners without injury. Schofield et al. 
(41) also reported 'uncontrollable anger' as an unresolved post injury symptom within $20 \%$ of their sample with TBI. Using both the PAI (62) and the BPAQ-SF (61) Diamond et al. (29) reported significantly higher aggression scores among their groups with TBI. Fishbein et al. (31) also reported significantly lower total aggression among their group without TBI when compared to those with past TBI.

For three studies, including Brewer-Smyth et al. (26) significantly higher rates of violent offences were recorded among offenders with past TBILOC. Pitman et al. (37) reported higher rates of violent offenses in their TBI group when compared to controls, while León-Carrion and Ramos (33) reported that the factor differentiating violent from non-violent prisoners was a history of untreated brain injuries.

\section{Psychiatric conditions}

Schofield et al. (42) included a measure of psychosis adapted from a previous study (63). They found that prisoners screening positive for psychosis were more likely to have sustained; any TBI, a TBI in the past year, or a TBILOC. Similarly, Ray et al. (38) identified a number of participants with psychiatric disorders who had completed a mental health screening tool provided by the prison. Of the inmates with TBI, $22 \%$ were also identified as having a psychiatric disorder compared to $9.9 \%$ of the sample without TBI. Finally, Slaughter et al. (43) reported a higher prevalence of psychiatric disorders in the group with TBI (84\%) when compared those without TBI (60\%) although this did not reach statistical significance.

\section{Additional findings}

Williams et al. (45) measured rates of repeat offenses reporting that participants with TBI were younger when first convicted of a criminal offense. This finding was further supported by Pitman et 
al. (37) who found a higher rate of past criminal conviction among prisoners with TBI. Similarly, Ray et al. (2014) reported that participants with TBI were 1.5 times more likely to have a prior arrest than those without.

Higher rates of health problems were also common among this population. Prisoners with TBI from the Pitman et al. (37) study reported significantly more health problems compared to the uninjured sample, as measured by the General Health Questionnaire (64). Walker et al. (44) also noted significantly higher rates of musculoskeletal, respiratory, circulatory, and skin problems among prisoners with TBI.

While Corrigan et al. (28) included a measure of sociopathy in their study no significant main or interactional effects were observed in their clusters. Despite this, there was a trend between the multiple mild untreated TBIs cluster and sociopathy.

Finally, both Merbitz et al. (34) and Shiroma et al. (22) focused on the rate of behavioural infractions committed by prisoners. Merbitz et al. (34) reported that significantly more disciplinary tickets (administered for each infraction of prison rules) were received by prisoners with TBI than those without. Shiroma et al. (22) further categorised prison behavioural infractions as either violent or non-violent comparing between participants with and without TBI for both their male and female groups. They found a significant association between behavioural infractions and TBI in males, along with an increased violent infraction rate in both males (86\%), and females (144\%) with TBI compared to those without.

\section{Comparison groups}

Of the twenty six studies, nine used comparison groups. Shiroma et al. (22), Pitman et al. (37), Merbitz et al. (34), and Suchy et al. (20) examined group differences between offenders with and 
without TBI. Ross and Hoaken (39) performed a comparative analysis between first time and return offenders while Brewer-Smyth et al. (26) and León-Carrión and Ramos (33) compared violent and non-violent offenders with TBI. Perkes et al. (36) explored TBI rates in both offenders and nonoffenders and Walker et al. (44) divided participants into one of three groups; no injury, one injury, and two or more injuries.

\section{Gender}

Nine studies included female prisoners in their analysis although only five of these provided significant detail to allow for gender comparison.

Fishbein et al. (31) reported TBI rates of $71.5 \%$ for women, compared to $64.1 \%$ for men. When LOC was controlled for these figures dropped to $47.5 \%$ and $41.9 \%$. Equally high rates of female TBI were observed in the study by Ferguson et al. (30) that reported $72 \%$ of the female prison sample had a past TBI versus $65 \%$ of the males. Shiroma et al. (22) also found the rate of medically attended TBI in females $(6.22 \%)$ to be higher than that of males $(5.65 \%)$, although their sample sizes varied dramatically (20,098 males; 1,512 females). In addition, the authors reported that while males were 5.88 times more likely to have received their injury while not incarcerated, females were 16.67 times more likely.

Colantonio et al. (27) reported both male and female rates of TBI with TBILOC. Of those who reported a past injury $62.7 \%$ were males and $37.3 \%$ were female, with the overall rate of TBILOC $50.4 \%$ for males and $38 \%$ for females. While Slaughter et al. (43) also included both male and female prisoners in their analysis, only 6 females consented to participate. All of these females reported past $T B I$, with five reporting they had sustained their injury in the past 12 months. Of the 63 males 
included, $85 \%$ reported a past TBI of which $31.7 \%(n=20)$ reported sustaining their injury in the past 12 months.

Diamond et al. (29) reported the number of past injuries for both males $(n=385)$ and females $(n=431)$ but failed to provide a breakdown of TBI by gender. Of the females with TBI, $45.4 \%$ reported LOC associated with their injuries, compared with $60 \%$ of the males. It should be noted that although no gender breakdown was provided, the 118 females interviewed reported a higher number of past injuries than the 107 males.

Brewer-Smyth et al. (26) was the only study to focus solely on female inmates, comparing 113 prisoners convicted of violent and non-violent crimes. They found $42 \%$ of the sample endorsed having at least one TBI with LOC and the majority of injuries were the result of violence perpetrated against the individual.

Finally, while both Bogner and Corrigan (25) and Corrigan et al. (28) included both male and female offenders in their analysis, no clear gender breakdown was provided. The remaining studies $(n=17)$ either only included male prisoners or failed to specify the gender of the inmates.

\section{Discussion}

\section{Rates of TBI}

Evident among the included studies were the varied methods for screening TBI. Of the 24 studies that assessed TBI rates only seven employed tools with proven reliability and validity. Within these seven studies variability in TBI rates remained high, ranging from $35.7 \%(38)$ to $88 \%(29)$. The number of items used in these measures may have had an effect on the resulting rate of reported TBI, with the abbreviated OSU-TBI-ID used by Ray et al. (38) reporting almost half the rate of the full measure. This shortened tool took 10 minutes to administer in comparison to the full tool which 
took between 60-120 minutes. This difference was also noted in both the Morrell et al. (35) and Merbitz et al. (34) studies where, following brief structured interviews, TBI rates of $24.9 \%$ and $31.7 \%$ were reported. It is possible that shorter screening tools may lack the depth of questioning required to delve into all past injuries, or it may be that longer measures provide participants with more time to remember previous TBls.

Evident while conducting this review was that varying definitions of $\mathrm{TBI}$, multiple approaches to data collection, as well as differences in the offender populations, complicate comparisons between studies making it difficult to ascertain the true rate of TBI within prison populations. The remaining nineteen included studies reported TBI rates ranging from $5.69 \%(22)$ to $87 \%(43)$. As such, there is a clear need for future studies to use valid and reliable measures in their screening of TBI. In addition to allowing researchers to pool their findings and establish definitive rates, a reliable and costeffective method of TBI screening would aid in the evaluation and monitoring of TBI by prison healthcare teams. Screening tools such as the BISI, TBIQ, and the OSU-TBI-ID, all offer reliable means of assessing past TBI in prisoner populations.

\section{Co-occurring problems}

Several studies highlighted the presence of TBI-related problems such as aggression, depression, substance use, psychiatric disorders, and neurocognitive deficits in their samples.

Particular focus was placed on the combined negative effects of TBI and drug use among prisoners. Several studies have highlighted a significant overlap between TBI and substance use $(23,29,31,36,42,44)$, and drug use is continually reported as a serious issue among all prisoners $(65,66)$. When compounded with TBI, significantly more complex impairments become evident $(44)$. Prisoners with TBI and comorbid drug abuse are at a significantly increased risk of depression, 
anxiety, and difficulties in regulating anger (44). It is important for prison healthcare teams to identify potential at risk prisoners so as to better tailor mental health and substance abuse interventions.

Prisoners with TBI are almost twice as likely to develop psychiatric disorders (38). With uncontrollable anger highlighted as an unresolved issue in many of these prisoners, there is support for the theory of a self-perpetuating cycle of trauma and distress among prisoners with co-occurring TBI and psychiatric illness (41). With the high degree of overlap between both conditions, effective screening and subsequent early intervention offer the potential to break this cycle and improve outcomes.

Suchy et al. (20) was the only study to focus solely on mTBI. No significant differences were observed between prisoners with mild injury and no-injury on traditional neuropsychological measures, though an increased novelty effect was noted among the group with mTBI . More importantly, the authors highlighted the lack of research into the compounding effects of multiple mild injuries. These subtle injuries are more likely to go un-noticed, especially if screening tools for TBI focus on LOC. As a result, prisoners may be less likely to report or even remember such occurrences despite research suggesting lasting abnormalities in white matter among such individuals $(67,68)$. It is important, therefore, not to dismiss mild injuries and there is a need for future research to compare the outcomes of prisoners with multiple mTBI and single more severe injuries.

From the included literature, it is evident that TBI is more than a single treatment issue. Rather, it presents alongside a complex array of impairments and difficulties, the extent and severity of which requires further exploration. The lack of research focusing on the effects of TBI on personality was also noted within the review. Factors such as empathy, narcissism, and self-monitoring behaviours have yet to be adequately addressed within this population. Additionally, despite the high degree of 
self-report measures employed, no study attempted to use qualitative methods to explore the experiences and beliefs of prisoners with regard to their injuries.

\section{TBI in female prisoners}

Studies including female prisoners were limited, and of the nine included in this review one sampled only 6 female participants (43), while three others provided no breakdown of the sample by gender $(25,28,29)$. Three studies reported higher rates of TBI among female prisoners in comparison to their male counterparts $(22,30,31)$. Fishbein et al. (31) also noted greater severity of drug use among female prisoners. Evident from the literature were differences in the cause of injury between males and females. Diamond et al. (29) reported that assault was the leading source of TBI for women (46.6\%), while vehicle related accidents caused the majority of injuries in males (35.6\%). BrewerSmyth et al. (26) similarly noted that most brain injuries sustained by their sample of 113 female prisoners were the result of violence perpetrated against them. It is likely that these gender differences are reflected in the differing needs of prisoners, with victims of assault likely to present with additional symptoms of Post-Traumatic Stress Disorder (PTSD) (69). It may also be possible that compounded mild injuries, caused by domestic abuse, produce more significant deficits in this population.

It was also observed that female prisoners are more likely to have had a TBI prior to their criminal offence than males $(22,27,30)$. The cause of this is unclear although it suggests that earlier interventions for young women with TBI may aid in reducing their risk of later criminal behaviour.

From the included literature it is clear that female prisoners are under-represented in the research. However, what was apparent among the included studies was that female prisoners with TBI present distinctly different needs to those of their male counterparts. This is reflected in higher rates of past 
sexual abuse (27), and reported ongoing symptoms of injury (30) among females compared to males. Future research is needed to expand on these differences as well as explore the variation in cause and nature of injuries sustained by women.

\section{Use of comparison groups}

Nine studies in our review included well described and controlled comparison groups. Of these, two compared violent and non-violent offenders $(26,33)$, four compared prisoners with and without TBI $(20,22,34,37)$, and the remaining three compared; first time and return inmates (39), inmates with differing numbers of injuries (44), and offenders and non-offenders (36).

There was a marked lack of comparison between prisoner samples with only three studies comparing different groups of prisoners $(26,33,39)$. Given the co-occurrence of TBI and aggression there is a need to further explore the differences in TBI among violent and non-violent prisoners. Currently, little more can be concluded than an association between TBI and violent crime, and further research is required to explore the nature of this association. Furthermore, the needs of prisoners can differ widely depending on their injury and future studies should examine the effectiveness of current rehabilitation programs for prisoners with varied severities of TBI.

While past studies have emphasised that combined TBI and low socioeconomic status is a contributing factor to criminal behaviour (41) little research has compared the outcomes of injuries in groups from differing backgrounds, such as urban and rural areas. It may be that early identification of injury and access to treatment factor into the relationship between TBI and offending behaviour.

Walker et al. (44) was the only study to compare groups based on severity of injury. By comparing injuries the authors were better able to categorise and assess the differing needs of each group. 
While participants with a single injury reported more alcohol problems and suicidal ideation than those with no injury, participants with two or more injuries reported more violent thoughts, cognitive processing problems, and lifetime symptoms of depression, than all other groups. Further research of this kind is needed to assess the differing needs of prisoners with varying severities of injury.

\section{Future research}

Comparison between studies is limited due to variability in both definitions of TBI and the screening methods employed. Further validation of the methods used to assess historic TBI is needed, to ensure consistency between research. Screening tools employed must also have proven validity and reliability to ensure accuracy in identification of TBI.

Emphasis has been placed on measures of aggression and anger, depression and anxiety, drug and alcohol use, and neuropsychological outcomes with a lack of investigation into factors such as personality, social functioning, perception, and communication. Future research is needed to address and explore the effect of TBI on these areas within prison populations.

No qualitative studies were found exploring the experiences of prisoners with TBI. Given that many prisoners were aware of their injuries, future research should aim to address the experiences and beliefs of these prisoners with regard to their injuries.

In the UK, the recent Bradley report (70) presented a comprehensive reform plan for mental illness and learning disability services in prisons. Despite widespread agreement that the needs of prisoners with TBI are poorly addressed, there is no research piloting potential interventions within UK prison settings. 
The lack of research on female prisoners with TBI is apparent. Brewer-Smyth et al. (26) was the only study to include a sample of solely female participants, observing that $42 \%$ reported at least one TBILOC. The authors went on to compare these rates to similar populations of women developing brain injuries as a result of domestic and physical abuse. While Fishbein et al. (31) and Ferguson et al. (30) both reiterated the correlation between abuse and TBI in women, their discussion was limited by the lack of available studies on the issue. Future research needs to explore and develop an understanding of the differing needs presented by female prisoners with TBI.

\section{Limitations}

This issue of definition was also apparent in the description of 'offenders' used by studies from different geographical regions. We chose to focus our review on studies of the general adult prison population, thus excluding research into specific sub-sets of this population such as elderly offenders, convicted murderers, or inmates on death row. Studies that failed to clearly define where their offenders were sourced from were excluded.

There was also a lack of geographic variation between included studies. Over half of the included studies were from either the USA $(n=14)$ or Australia $(n=5)$, while the remainder were conducted in Canada, UK, New Zealand, and Spain. Given the variation in culture, government policy, and criminal justice systems in these countries, it is difficult to produce generalisable conclusions about prisoners with TBI. Without detailed socio-demographic characteristics and offending histories, comparison between prisoners across international contexts is limited. In addition, our review lacked the resources to include studies that were not published in English, further limiting the number of studies included.

\section{Conclusions}


Authors must consider the validity and comparability of their measures, along with the generalisability of their studies. Clearly defined terms, adequate participant information, and substantial detail on the research setting are all necessary for future research endeavours. Only by adhering to such guidelines can the external validity of studies be ensured.

TBI presents as a serious health risk for prisoners. Several co-occurring factors were identified in this review, including aggression, drug abuse, depression, and neurocognitive deficits. Current research suggests there is justification for full TBI screening and assessment upon entry into prison. Furthermore, an assessment of resources and available interventions within the prison service is required to ensure that prisoners identified as having a TBI can access and avail of support services.

\section{References}

1. Thurman DJ, Alverson C, Dunn KA, Guerrero J SJ. Traumatic brain injury in the United States: a public health perspective. J Head Trauma Rehabil. 1999;14(6):602-15.

2. Faul M, et al. Traumatic brain injury in the United States: emergency department visits, hospitalizations and deaths 2002-2006. Centers for Disease Control and Prevention, National Center for Injury Prevention and Control; 2010.

3. Hillier S, Hiller J MJ. Epidemiology of traumatic brain injury in South Australia. Brain Inj. 1997;11:649-59.

4. Tagliaferri F, Compagnone C, Korsic M, Servadei F, Kraus J. A systematic review of brain injury epidemiology in Europe. Acta Neurochir (Wien). 2006;148(3):255-67.

5. Escorpizo R, Brage S, Homa D, Stucki G. Handbook of vocational rehabilitation and disability evaluation: application and implementation of the ICF. Springer.; 2014. p 263-70. 
6. Crothers IR, Linden M, Kennedy N. Attitudes of children towards peers with acquired brain injury (ABI). Brain Inj. 2007;21(1):47-52.

7. Morton MV, Wehman P. Psychosocial and emotional sequelae of individuals with traumatic brain injury: a literature review and recommendations. Brain Inj. 1995;9(1):81-92.

8. Prigatano GP. Personality disturbances associated with traumatic brain injury. J Consult Clin Psychol. 1992;60(3):360.

9. Hoofien D, Gilboa A, Vakil E, Donovick PJ. Traumatic brain injury (TBI) 10-20 years later: a comprehensive outcome study of psychiatric symptomatology, cognitive abilities and psychosocial functioning. Brain Inj. 2001;15(3):189-209.

10. Rosema S, Crowe L, Anderson V. Social function in children and adolescents after traumatic brain injury: a systematic review 1989-2011. J Neurotrauma. 2012;29(7):1277-91.

11. Kim E. Agitation, aggression, and disinhibition syndromes after traumatic brain injury. NeuroRehabilitation. 2002;17(4):297-310.

12. Baguley IJ, Cooper J, Felmingham K. Aggressive behavior following traumatic brain injury: how common is common? J Head Trauma Rehabil. 2006;21(1):45-56.

13. Ogilvie JM, Stewart AL, Chan RCK, Shum DHK. Neuropsychological measures of executive function and antisocial behavior: a meta-analysis. Criminology. 2011;49(4):1063-107.

14. Bruns J, Hauser WA. The epidemiology of traumatic brain injury: a review. Epilepsia. 2003;44(s10):2-10.

15. Elbogen EB, Wolfe JR, Cueva M, Sullivan C JJ. Longitudinal predictors of criminal arrest after traumatic brain injury: results from the traumatic brain injury model system national database. J Head Trauma Rehabil. 2014. 
16. Shiroma EJ, Ferguson PL, Pickelsimer EE. Prevalence of traumatic brain injury in an offender population: a meta-analysis. J Correct Health Care. 2010;16(2):147-59.

17. Farrer TJ, Frost RB, Hedges DW. Prevalence of traumatic brain injury in juvenile offenders: a meta-analysis. Child Neuropsychol. 2013;19(3):225-34.

18. Hughes N, Williams WH, Chitsabesan P, Walesby RC, Mounce LT, Clasby B. The prevalence of traumatic brain injury among young offenders in custody. J Head Trauma Rehabil. 2015;30(2):94-105.

19. National Institute of Health. Quality assessment tool for observational cohort and crosssectional studies. http://www.nhlbi.nih.gov/health-pro/guidelines/in-develop/cardiovascularrisk-reduction/tools/cohort. Accessed 2015, February 02.

20. Suchy Y, Euler M, Eastvold A. Exaggerated reaction to novelty as a subclinical consequence of mild traumatic brain injury. Brain Inj. 2014;28(7):972-9.

21. Templer DI, Kasiraj J, Trent NH, Trent A, Hughey B, Keller WJ, Orling RA, Thomas-Dobson S. Exploration of head injury without medical attention. Percept Mot Skills. 1992;75(1):195-202.

22. Shiroma EJ, Pickelsimer EE, Ferguson PL, Gebregziabher M, Lattimore PK, Nicholas JS, Dukes T, Hunt KJ. Association of medically attended traumatic brain injury and in-prison behavioral infractions: a statewide longitudinal study. J Correct Heal Care. 2010;16(4):273-86.

23. Barnfield TV, Leathem JM. Incidence and outcomes of traumatic brain injury and substance abuse in a New Zealand prison population. Brain Inj. 1998;12(6):455-66.

24. Barnfield TV, Leathem JM. Neuropsychological outcomes of traumatic brain injury and substance abuse in a New Zealand prison population. Brain Inj. 1998;12(11):951-62. 
25. Bogner J, Corrigan JD. Reliability and predictive validity of the Ohio State University TBI identification method with prisoners. J Head Trauma Rehabil. 2009;24(4):279-91.

26. Brewer-Smyth K, Burgess AW, Shults J. Physical and sexual abuse, salivary cortisol, and neurologic correlates of violent criminal behavior in female prison inmates. Biol Psychiatry. 2004;55(03):21-31.

27. Colantonio A, Kim H, Allen S, Asbridge M, Petgrave J, Brochu S. Traumatic brain injury and early life experiences among men and women in a prison population. J Correct Health Care. 2014;20(4):271-9.

28. Corrigan JD, Bogner J, Holloman C. Lifetime history of traumatic brain injury among persons with substance use disorders. Brain Inj. 2012;26(2):139-50.

29. Diamond PM, Harzke AJ, Magaletta PR, Cummins AG, Frankowski R. Screening for traumatic brain injury in an offender sample: a first look at the reliability and validity of the Traumatic Brain Injury Questionnaire. J Head Trauma Rehabil. 2007;22(6):330-8.

30. Ferguson PL, Pickelsimer EE, Corrigan JD, Bogner JA, Wald M. Prevalence of traumatic brain injury among prisoners in South Carolina. J Head Trauma Rehabil. 2012;27(3):E11-20.

31. Fishbein D, Dariotis JK, Ferguson PL, Pickelsimer EE. Relationships between traumatic brain injury and illicit drug use and their association with aggression in inmates. Int J Offender Ther Comp Criminol. 2014 Oct; 0306624X14554778.

32. Kavanagh L, Rowe D, Hersch J, Barnett KJ, Reznik R. Neurocognitive deficits and psychiatric disorders in a NSW prison population. Int J Law Psychiatry. 2010;33(1):20-6. 
33. León-Carrión J, Ramos FJC. Blows to the head during development can predispose to violent criminal behaviour: rehabilitation of consequences of head injury is a measure for crime prevention. Brain Inj. 2003;17:207-16.

34. Merbitz C, Jain S, Good GL, Jain A. Reported head injury and disciplinary rule infractions in prison. J of Offender Rehabilitation. 1995;22(3-4):37-41.

35. Morrell RF, Merbitz CT, Jain S, Jain S. Traumatic brain injury in prisoners. J of Offender Rehabilitation. 1998;27(3-4):37-41.

36. Perkes I, Schofield PW, Butler T, Hollis SJ. Traumatic brain injury rates and sequelae: a comparison of prisoners with a matched community sample in Australia. Brain Inj. 2011;25(2):131-41.

37. Pitman I, Haddlesey C, Ramos SDS, Oddy M, Fortescue D. The association between neuropsychological performance and self-reported traumatic brain injury in a sample of adult male prisoners in the UK. Neuropsychol Rehabil. 2014.

38. Ray B, Sapp D, Kincaid A. Traumatic brain injury among Indiana state prisoners. J Forensic Sci. 2014;59(5):1248-53.

39. Ross EH, Hoaken PNS. Executive cognitive functioning abilities of male first time and return Canadian federal inmates. Can J Criminol Crim Justice. 2011;53(4):377-403.

40. Schofield P, Butler T, Hollis S, D’Este C. Are prisoners reliable survey respondents? A validation of self-reported traumatic brain injury (TBI) against hospital medical records. Brain Inj . 2011;25(1):74-82.

41. Schofield PW, Butler TG, Hollis SJ, Smith NE, Lee SJ, Kelso WM. Traumatic brain injury among Australian prisoners: rates, recurrence and sequelae. Brain Inj. 2006;20(5):499-506. 
42. Schofield PW, Butler TG, Hollis SJ, Smith NE, Lee SJ, Kelso WM. Neuropsychiatric correlates of traumatic brain injury (TBI) among Australian prison entrantst. Brain Inj. 2006:1409-18.

43. Slaughter B, Fann JR, Ehde D. Traumatic brain injury in a county jail population: prevalence, neuropsychological functioning and psychiatric disorders. Brain Inj. 2003;17(9):731-41.

44. Walker R, Hiller M, Staton M, Leukefeld CG. Head injury among drug abusers: an indicator of co-occurring problems. J Psychoactive Drugs. 2003;35:343-53.

45. Williams WH, Mewse AJ, Tonks J, Mills S, Burgess CNW, Cordan G. Traumatic brain injury in a prison population: prevalence and risk for re-offending. Brain Inj. 2010;24(10):1184-8.

46. Kay T, Harrington DE, Adams R. American Congress of Rehabilitation Medicine, Head Injury Interdisciplinary Special Interest Group. Definition of mild traumatic brain injury. J Head Trauma Rehabil. 1993;8(3):86-7.

47. Wechsler D. Wechsler Abbreviated Scale of Intelligence. 2nd Edn. Oxford: Pearson Assessment. 2011.

48. Wilson BA, Evans JJ, Alderman N, Burgess PW, Emslie H. Behavioural assessment of the dysexecutive syndrome. Methodol Front Exec Funct. 1997;239-50.

49. Randolph C, Tierney MC, Mohr E, Chase TN. The Repeatable Battery for the Assessment of Neuropsychological Status (RBANS): preliminary clinical validity. J Clin Exp Neuropsychol. 1998;20(3):310-9.

50. Wechsler D. Wechsler Adult Intelligence Scale. 3rd ed. San Antonio, TX Psychol Corp. 1997.

51. Bergner M, Bobbitt RA, Carter WB GB. The Sickness Impact Profile: development and final revision of a health status measure. Med Care. 1981;(19):787-805. 
52. Grace J MP. Frontal Systems Behavioral Scale Professional Manual. Odessa, FL Psychol Assess Resour Inc. 2001.

53. Delis D, Kaplan E KJDK. Executive Function System, Examiner's Manual. San Antonio, TX Psychol Corp. 2001.

54. Zachary RA, Paulson MJ GR. Estimating WAIS IQ from the Shipley Institute of Living Scale using continuously adjusted age norms. J Clin Psychol. 1985;(41):820-31.

55. Suchy Y, Derbidge C CC. Behavioral Dyscontrol Scale- Electronic Version: First examination of reliability, validity, and incremental utility. Clin Neuropsychol. 2005;(19):4-26.

56. McLellan AT, Kushner H, Metzger D, Peters R, Smith I, Grissom G, Pettinati H, Argeriou M. The fifth edition of the Addiction Severity Index. J Subst Abus Treat. 1992;9(3):119-213.

57. Saunders JB, Aasland OG BT. Development of the alcohol use disorders identification test (AUDIT): WHO collaborative project on early detection of persons with harmful alcohol consumption. Addiction. 1993;(88):791-804.

58. Teplin LA SJ. Screening for severe mental disorder in jails: the development of the referral decision scale. Law Hum Behav. 1989;13(1):1-18.

59. Beck AT, Epstein N, Brown G, Steer RA. An inventory for measuring clinical anxiety: psychometric properties. J Consult Clin Psychol. 1988;56(6):893.

60. Beck AT, Steer RA, Carbin MG. Psychometric properties of the Beck Depression Inventory: twenty-five years of evaluation. Clin Psychol Rev. 1988;8(1):77-100.

61. Buss AH, Perry M. The aggression questionnaire. Journal of personality and social psychology. 1992;63(3):452.

62. Morey LC. Personality Assessment Inventory. Odessa, Fla Psychol Assess Resour Inc. 1991. 
63. Jablensky A, McGrath J, Herrman H, Castle D, Gureje O, Evans M, Carr V, Morgan V, Korten A, Harvey C. Psychotic disorders in urban areas: an overview of the Study on Low Prevalence Disorders. Aust N Z J Psychiatry. 2000;34(2):221-36.

64. Goldberg DP, Hillier VF. A scaled version of the General Health Questionnaire. Psychol Med. 1979;9(01):139-45.

65. Karberg JC JD. Substance dependence, abuse, and treatment of jail inmates, 2002. Dept Justice Publ NCJ. 2005;209588(Washington, DC: Office of Justice Programs, Bureau of Justice Statistics).

66. Mumola CJ KJ. Drug use and dependence, state and federal prisoners, 2004. Dept Justice Publ. 2006; Washington(NCJ 213530.).

67. Aoki Y, Inokuchi R, Gunshin M, Yahagi N SH. Diffusion tensor imaging studies of mild traumatic brain injury: a meta-analysis. J Neurol Neurosurg Psychiatry. 2012;(83):870-6.

68. Shenton ME, Hamoda HM, Schneiderman JS, Bouix S, Pasternak O, Rathi Y, Vu MA, Purohit MP, Helmer K, Koerte I et al. A review of magnetic resonance imaging and diffusion tensor imaging findings in mild traumatic brain injury. Brain Imaging Behav. 2012;(6):137-92.

69. Yehuda R, Schmeidler J, Siever L, Binder-Brynes K, Elkin A. Individual differences in posttraumatic stress disorder symptom profiles in Holocaust survivors in concentration camps or in hiding. J Trauma Stress. 1997 Jul;10(3):453-63.

70. Bradley KJCB. The Bradley Report: Lord Bradley's review of people with mental health problems or learning disabilities in the criminal justice system. London Dep Heal. 2009. 